Cook Emma S (Orcid ID: 0000-0002-4552-9747)

Dand Nick (Orcid ID: 0000-0002-1805-6278)

Yiu Zenas Zee Ngai (Orcid ID: 0000-0002-1831-074X)

Bachelez Herve (Orcid ID: 0000-0002-2845-4384)

Capon Francesca (Orcid ID: 0000-0003-2432-5793)

Contreras Claudia (Orcid ID: 0000-0002-1465-5126)

Di Meglio Paola (Orcid ID: 0000-0002-2066-7780)

Gisondi Paolo (Orcid ID: 0000-0002-1777-9001)

Naldi Luigi (Orcid ID: 0000-0002-3160-2835)

Puig Lluís (Orcid ID: 0000-0001-6083-0952)

Griffiths Christopher Ernest, Maitland (Orcid ID: 0000-0001-5371-4427)

\title{
Vaccine hesitancy and access to psoriasis care in the COVID-19 pandemic: findings from a global patient-reported cross-sectional survey
}

\section{Dear Editor,}

COVID-19 vaccines protect against severe COVID-19 outcomes, however many individuals remain unvaccinated ${ }^{1,2}$. Vaccine hesitancy (delayed acceptance/refusal of vaccination despite service availability) threatens public health. In the UK general population, vaccine hesitancy is higher in women, younger age and ethnic minority groups ${ }^{3,4}$. Individuals with psoriasis, particularly those taking systemic immunosuppressants, are prioritised for COVID-19 vaccination $^{5}$. However, information on vaccine hesitancy and its contributing factors in psoriasis patients is scarce ${ }^{6}$.

We used global patient-reported PsoProtectMe survey data ${ }^{7}$ to explore the impact of organisational and individual factors on COVID-19 vaccine hesitancy. Data from 802 individuals with psoriasis from 89 countries were available (extracted 09/08/2021). 322 (40.1\%) reported disrupted access to psoriasis care. These individuals were younger (median age 44 [IQR 33-56] versus 54 [IQR 42-64]) and more likely to be of non-white ethnicity (13.8\% versus $10.2 \%$ ). They had a shorter psoriasis duration (median 23 years [IQR 10-36] versus 31 years [IQR 17-44]) and more severe psoriasis (6.1\% versus $2.8 \%)$. The proportion of participants taking systemic immunosuppressants was similar between those with and without disrupted access to care, but a smaller proportion with disrupted care were taking targeted immunosuppressants than those without disruption (72/131 [55.0\%] versus 140/194 [72.1\%]).

$611(80.9 \%)$ of 755 participants had received at least 1 vaccine dose. Sixty-three $(8.3 \%)$ were vaccine hesitant. These individuals were younger (median age 36 [IQR 30-50] versus 52 [IQR 39-63]), more likely to be of non-white ethnicity (20.0\% versus $7.2 \%$ ), live outside the UK (13.6\% versus 5.1\%), have a numerically lower BMI (median $24.5 \mathrm{~kg} / \mathrm{m}^{2}$ [IQR $21.7-28.3$ ] versus $26.5 \mathrm{~kg} / \mathrm{m}^{2}$ [23.2-30.9]) and a shorter disease duration (median 19 years [IQR 9-32]

This article has been accepted for publication and undergone full peer review but has not been through the copyediting, typesetting, pagination and proofreading process which may lead to differences between this version and the Version of Record. Please cite this article as doi: $10.1111 /$ bjd. 21042

This article is protected by copyright. All rights reserved. 
versus 28 years [IQR 14-42]). They were less likely to be taking systemic immunosuppressants (26.0\% versus $45.3 \%)$. The commonest reasons for hesitancy were concerns regarding sideeffects, the vaccine being new and psoriasis worsening post-vaccination.

In an unadjusted logistic regression model, strongly agreeing that psoriasis care was disrupted associated with vaccine hesitancy compared to strongly disagreeing (OR 2.97, 95\%CI 1.237.13, $\mathrm{p}=0.015)$. The direction of association remained after adjusting for age, sex, ethnicity, although was not statistically significant (adjOR 1.90, 95\%CI 0.72-5.05) (Fig.1). In the imputed multivariate model (fitted due to missing demographic data), the association was stronger but non-significant (adjOR 2.32, 95\%CI 0.94-5.71). 56/320 (17.5\%) individuals taking standard/targeted/combination immunosuppressants were non-adherent. Non-adherence and vaccine hesitancy were not significantly associated (adjOR 2.96, 95\% CI 0.77-11.3).

We observe an association between disrupted access to psoriasis care and COVID-19 vaccine hesitancy, partly mediated by confounding demographic variables. Individuals feeling disenfranchised by healthcare services were more likely to be vaccine hesitant. In support, higher vaccine hesitancy in the general population is observed in those with negative experiences of healthcare and negative perceptions of doctors ${ }^{4}$. Higher care expectations, sometimes seen in individuals with worse disease ${ }^{8}$, may also be contributory. Patients taking targeted immunosuppressants were less likely to report disrupted access to care, possibly due to more frequent monitoring in secondary care, which may have been prioritised during the pandemic.

A minority (8\%) of our sample reported vaccine hesitancy. These findings support current limited data on vaccine hesitancy amongst psoriasis patients ${ }^{6}$. In contrast to a prior report in psoriasis $^{6}$, but in keeping with general population trends ${ }^{4}$, hesitancy was more prominent amongst younger people. Our study was conducted after the COVID-19 vaccine roll-out commenced, addressing a limitation of studies characterising intention rather than actual behaviour ${ }^{6}$. In keeping with other studies, our findings also indicate greater hesitancy in individuals of non-white ethnicity, however there were no gender differences ${ }^{4}$.

We are unable to directly compare our global psoriasis dataset to the general population/other diseases due to a lack of control samples. Participants were mostly from UK, female and of white ethnicity, limiting generalisability. Proportionally more patients reported receiving 
targeted versus standard immunosuppression, indicating ascertainment bias. Directions of associations cannot be definitively ascertained due to the cross-sectional study design. Impacts on care and/or vaccine uptake may have been underestimated since individuals participating in health surveys may be more engaged with healthcare/vaccination services. PsoProtectMe was updated one year following its launch to include COVID-19 vaccine hesitancy/access to care questions, hence the current sample may not be representative of the original larger sample ${ }^{7}$.

Taken together, these data indicating that a minority of individuals with psoriasis have vaccine hesitancy are promising for current/future COVID-19 booster vaccine uptake. Identifying individuals who are disenfranchised by healthcare services and addressing their concerns regarding COVID-19 vaccination will help mitigate risks from the ongoing pandemic.

Acknowledgments: We are extremely grateful to all the patients who have contributed to PsoProtectMe, the professional and patient organizations who supported or promoted PsoProtectMe, and Prof Lars Iversen, Prof Nick Reynolds and Prof Joel Gelfand for their valuable input. We would like to acknowledge the following individuals for help with translating the PsoProtectMe survey; Dr Haleema Alfailakawi, Dr Wisam Alwan, Dr Rosa Andres Ejarque, Dr Ines Barbosa, Ms Carmen Bugarin Diz, Ms Katarzyna Grys, Dr Mahira Hamdy El Sayed, Mr Tran Hong Truong, Mr Masanori Okuse, Ms Dagmara Samselska, Ms Isabella Tosi, Ms Ya-Hsin Wang. Thank you to the Engine Group UK for their generous creative input and website expertise.

Katie Bechman, ${ }^{1 *}$ Emma S. Cook, ${ }^{1 *}$ Nick Dand, ${ }^{2,3}$ Zenas Z.N. Yiu, ${ }^{4}$ Teresa Tsakok, ${ }^{5}$ Freya Meynell, ${ }^{5}$ Bolaji Coker, ${ }^{6}$ Alexandra Vincent, ${ }^{6}$ Herve Bachelez, ${ }^{7,8}$ Ines Barbosa, ${ }^{5}$ Matthew A. Brown, ${ }^{1,6}$ Francesca Capon, ${ }^{2,6}$ Claudia R. Contreras, ${ }^{9}$ Claudia De La Cruz, ${ }^{10}$ Paola Di Meglio, ${ }^{6,11}$ Paolo Gisondi, ${ }^{12}$ Denis Jullien, ${ }^{13,14}$ Jade Kelly, ${ }^{4}$ Jo Lambert, ${ }^{15}$ Camille Lancelot, ${ }^{16}$ Sinead M. Langan, ${ }^{5,17}$ K.J. Mason,,${ }^{4,18}$ Helen McAteer, ${ }^{19}$ Lucy Moorhead, ${ }^{5}$ Luigi Naldi, ${ }^{20}$ Sam Norton, ${ }^{21}$ Lluís Puig, ${ }^{22}$ Phyllis I. Spuls, ${ }^{23}$ Tiago Torres, ${ }^{24}$ Dominic Urmston, ${ }^{19}$ Amber Vesty, ${ }^{19}$ Richard B. Warren, ${ }^{4}$ Hoseah Waweru, ${ }^{16}$ John Weinman, ${ }^{25}$ Christopher M. Griffiths, ${ }^{4}$ Jonathan N. Barker, ${ }^{6,11}$ Catherine H. Smith, ${ }^{5,6}$ James B. Galloway ${ }^{1 \dagger}$ and Satveer K. Mahil ${ }^{5,6 \dagger}$

\section{On behalf of the PsoProtect study group}


${ }^{1}$ Centre for Rheumatic Diseases, King's College London, London, UK; ${ }^{2}$ Department of Medical and Molecular Genetics, School of Basic and Medical Biosciences, Faculty of Life Sciences and Medicine, King's College London, London, UK; ${ }^{3}$ Health Data Research UK, London, UK; ${ }^{4}$ Dermatology Centre, Salford Royal NHS Foundation Trust, The University of Manchester, Manchester Academic Health Science Centre, NIHR Manchester Biomedical Research Centre, Manchester, UK; ${ }^{5}$ St John's Institute of Dermatology, Guy's and St Thomas' NHS Foundation Trust and King's College London, London, UK; ${ }^{6} \mathrm{NIHR}$ Biomedical Research Centre at Guy's and St Thomas' NHS Foundation Trust and King's College London, London, UK; ${ }^{7}$ Department of Dermatology, AP-HP Hôpital Saint-Louis, Paris, France; ${ }^{8}$ INSERM U1163, Imagine Institute for Human Genetic Diseases, Université de Paris, Paris, France; ${ }^{9}$ Catedra de Dermatologia, Hospital de Clinicas, Facultad de Ciencias Medicas, Universidad Nacional de Asuncion, Paraguay; ${ }^{10}$ Clinica Dermacross, Santiago, Chile; ${ }^{11} \mathrm{St}$ John's Institute of Dermatology, School of Basic \& Medical Biosciences, Faculty of Life Sciences \& Medicine, King's College London, London, UK; ${ }^{12}$ Section of Dermatology and Venereology, University of Verona, Verona, Italy; ${ }^{13}$ Department of Dermatology, Edouard Herriot Hospital, Hospices Civils de Lyon, University of Lyon, Lyon, France; ${ }^{14}$ Groupe de recherche sur le psoriasis (GrPso) de la Société française de dermatologie, Paris, France; ${ }^{15}$ Department of Dermatology, Ghent University, Ghent, Belgium; ${ }^{16}$ International Federation of Psoriasis Associations; ${ }^{17}$ Faculty of Epidemiology, and Population Health, London School of Hygiene and Tropical Medicine, London, UK; ${ }^{18}$ School of Medicine, Keele University, Keele, UK; ${ }^{19}$ The Psoriasis Association, Northampton, UK; ${ }^{20}$ Centro Studi GISED, Bergamo, Italy; ${ }^{21}$ Psychology Department, Institute of Psychiatry, Psychology and Neuroscience, King's College London, UK; ${ }^{22}$ Department of Dermatology, Hospital de la Santa Creu i Sant Pau, Universitat Autònoma de Barcelona, Barcelona, Catalonia, Spain; ${ }^{23}$ Department of Dermatology, Amsterdam Public Health/Infection and Immunology, Amsterdam University Medical Centers, Location AMC, Amsterdam, The Netherlands; ${ }^{24}$ Department of Dermatology, Centro Hospitalar do Porto, Portugal; ${ }^{25}$ School of Cancer and Pharmaceutical Sciences, King's College London, London, UK.

*Joint first authors

†Joint senior authors

\section{Correspondence: Dr Satveer Mahil}

Email: satveer.mahil@kcl.ac.uk 


\section{ORCiD:}

http://orcid.org/0000-0002-4552-9747 (ES Cook)

http://orcid.org/0000-0002-1805-6278 (N Dand)

http://orcid.org/0000-0002-1831-074X (ZZN Yiu)

https://orcid.org/0000-0003-1895-6070 (T Tsakok)

http://orcid.org/0000-0002-2845-4384 (H Bachelez)

https://orcid.org/0000-0003-0538-8211 (MA Brown)

http://orcid.org/0000-0003-2432-5793 (F Capon)

https://orcid.org/0000-0002-8925-0457 (C De la Cruz)

http://orcid.org/0000-0002-2066-7780 (P Di Meglio)

http://orcid.org/0000-0002-1777-9001 (P Gisondi)

https://orcid.org/0000-0002-2995-7928 (D Jullien)

https://orcid.org/0000-0001-5303-9310 (J Lambert)

https://orcid.org/0000-0002-7022-7441 (SM Langan)

https://orcid.org/0000-0002-2887-5533 (H McAteer)

http://orcid.org/0000-0002-3160-2835 (L Naldi)

https://orcid.org/0000-0003-1714-9963 (S Norton)

http://orcid.org/0000-0001-6083-0952 (L Puig)

https://orcid.org/0000-0002-6035-2863 (P Spuls)

https://orcid.org/0000-0003-0404-0870 (T Torres)

https://orcid.org/0000-0002-2918-6481 (RB Warren)

https://orcid.org/0000-0002-6786-0166 (J Weinman)

http://orcid.org/0000-0001-5371-4427 (CM Griffiths)

https://orcid.org/0000-0002-9030-183X (JN Barker)

https://orcid.org/0000-0001-9918-1144 (CH Smith)

https://orcid.org/0000-0002-1230-2781 (JB Galloway)

https://orcid.org/0000-0003-4692-3794 (SK Mahil)

\section{References}

1 Rotshild V, Hirsh-Raccah B, Miskin I et al. Comparing the clinical efficacy of COVID-19 vaccines: a systematic review and network meta-analysis. Sci Rep. 2021 Dec; 11(1):22777. 
2 Ritchie H, Mathieu E, Rodés-Guirao L et al. Coronavirus Pandemic (COVID-19) Vaccinations. 2020. Available at: https://ourworldindata.org/covid-vaccinations (last accessed 6 January 2022).

3 MacDonald NE. Vaccine hesitancy: Definition, scope and determinants. Vaccine. 2015 Aug; 33(34):4161-4.

4 Freeman D, Loe BS, Chadwick A et al. COVID-19 vaccine hesitancy in the UK: the Oxford coronavirus explanations, attitudes, and narratives survey (Oceans) II. Psychol Med. 2020 Dec; 11:1-15.

5 Salisbury D, Ramsay M, Noakes K. Chapter 14a - COVID-19 - SARS-CoV-2. In: Immunisations Against Infectious Disease (Green Book). Department of Health; 2021. 6 Sotiriou E, Bakirtzi K, Papadimitriou I et al. COVID-19 vaccination intention among patients with psoriasis compared with immunosuppressed patients with other skin diseases and factors influencing their decision. Br J Dermatol. $2021 \mathrm{Jul} ;$ 185(1):209-10.

7 Mahil SK, Yates M, Langan SM et al. Risk-mi tigating behaviours in people with inflammatory skin and joint disease during the COVID-19 pandemic differ by treatment type: a cross-sectional patient survey*. Br J Dermatol. 2021 Jul; 185(1):80-90.

8 Bhutani T, Wong JW, Bebo BF et al. Access to health care in patients with psoriasis and psoriatic arthritis: data from National Psoriasis Foundation survey panels. JAMA Dermatol. 2013 Jun; 149(6):717-21.

Funding sources: We acknowledge financial support from the Department of Health via the National Institute for Health Research (NIHR) Biomedical Research Centre based at Guy's and St Thomas' NHS Foundation Trust and King's College London, the NIHR Manchester Biomedical Research Centre and the Psoriasis Association. The views expressed are those of the author(s) and not necessarily those of the NHS, the NIHR, or the Department of Health and Social Care. SKM is funded by a Medical Research Council (MRC) Clinical Academic Research Partnership award (MR/T02383X/1). ND is funded by Health Data Research UK (MR/S003126/1), which is funded by the UK MRC, Engineering and Physical Sciences Research Council; Economic and Social Research Council; Department of Health \& Social Care (England); Chief Scientist Office of the Scottish Government Health and Social Care Directorates; Health and Social Care Research and Development Division (Welsh Government); Public Health Agency (Northern Ireland); British Heart Foundation; and Wellcome Trust. ZZNY is funded by a NIHR Academic Clinical Lectureship through the 
University of Manchester. CEMG is a NIHR Emeritus Senior Investigator and is funded in part by the MRC (MR/101 1808/1). CEMG and RBW are in part supported by the NIHR Manchester Biomedical Research Centre. SML is supported by a Wellcome senior research fellowship in clinical science $(205039 / \mathrm{Z} / 16 / \mathrm{Z})$; this research was funded in whole or in part by the Wellcome Trust [205039/Z/16/Z]. For the purpose of Open Access, the author has applied a CC BY public copyright licence to any Author Accepted Manuscript (AAM) version arising from this submission. SML is also supported by Health Data Research UK (grant no. LOND1), which is funded by the UK MRC, Engineering and Physical Sciences Research Council, Economic and Social Research Council, Department of Health and Social Care (England), Chief Scientist Office of the Scottish Government Health and Social Care Directorates, Health and Social Care Research and Development Division (Welsh Government), Public Health Agency (Northern Ireland), British Heart Foundation and Wellcome Trust.

\section{Conflicts of interest:}

Nothing to disclose: Dr Bechman, Ms Cook, Dr Dand, Prof. Langan, Dr. Norton, Dr. Tsakok, Dr. Yiu, Dr De La Cruz, Dr. Contreras, Ms. Vesty, Ms. Vincent, Mr. Bola Coker, Ms. Meynell, Ms Kelly, Ms Lancelot, Dr. Lambert, Prof. Brown, Prof. Naldi.

Prof. Barker reports grants and personal fees from Abbvie, grants and personal fees from Novartis, grants and personal fees from Lilly, grants and personal fees from J\&J, from null, during the conduct of the study.

Prof. Griffiths reports grants and personal fees from AbbVie, grants from Amgen, grants from BMS, grants and personal fees from Janssen, grants from LEO, grants and personal fees from Novartis, grants from Pfizer, grants from Almirall, grants and personal fees from Lilly, grants and personal fees from UCB Pharma, outside the submitted work.

Prof. Jullien reports personal fees and non-financial support from Abbvie, personal fees and non-financial support from Novartis, personal fees and non-financial support from JanssenCilag, personal fees and non-financial support from Lilly, personal fees and non-financial support from Leo-Pharma, personal fees and non-financial support from MEDAC, personal fees and non-financial support from Celgene, personal fees from Amgen, outside the submitted work.

Dr. Capon reports consultancy fees from AnaptysBio, grants from Boheringer-Ingelheim, outside the submitted work.

Prof. Bachelez reports personal fees from Abbvie, personal fees from Janssen, personal fees from LEO Pharma, personal fees from Novartis, personal fees from UCB, personal fees from 
Almirall, personal fees from Biocad, personal fees from Boehringer-Ingelheim, personal fees from Kyowa Kirin, personal fees from Pfizer, outside the submitted work.

Prof. Gisondi reports personal fees from Abbvie, Amgen, Eli Lilly, Janssen, Novartis, Pierre Fabre, Sandoz, UCB, outside the submitted work.

Dr. Galloway reports personal fees from Abbvie, personal fees from Sanofi, personal fees from Novartis, personal fees from Pfizer, grants from Eli Lilly, personal fees from Janssen, personal fees from UCB, outside the submitted work.

Prof. Weinmann has presented talks for Abbvie, Abbott, Bayer, Chiesi, Boehringer Ingelheim, Roche and Merck.

Dr. Mason reports personal fees from LEO Pharma and Novartis, outside the submitted work. Ms. Moorhead reports personal fees from Abbvie, personal fees from Celgene, personal fees from Janssen, personal fees from LEO Pharma, personal fees from Novartis, personal fees from UCB, outside the submitted work.

Dr. Puig reports grants and personal fees from AbbVie, grants and personal fees from Almirall, grants and personal fees from Amgen, grants and personal fees from Boehringer Ingelheim, personal fees from Bristol Myers Squibb, personal fees from Fresenius-Kabi, grants and personal fees from Janssen, grants and personal fees from Lilly, personal fees from Mylan, grants and personal fees from Novartis, personal fees from Pfizer, personal fees from Sandoz, personal fees from Sanofi, personal fees from Samsung-Bioepis, grants and personal fees from UCB, outside the submitted work.

Dr. Mahil reports departmental income from Abbvie, Almirall, Eli Lilly, Janssen-Cilag, Novartis, Sanofi, UCB, outside the submitted work.

Dr. Di Meglio reports grants and personal fees from UCB, personal fees from Novartis, personal fees from Janssen, outside the submitted work.

Prof. Warren reports grants and personal fees from Abbvie, grants and personal fees from Celgene, grants and personal fees from Eli Lilly, grants and personal fees from Novartis, personal fees from Sanofi, grants and personal fees from UCB|, grants and personal fees from Almirall, grants and personal fees from Amgen, grants and personal fees from Janssen, grants and personal fees from Leo, grants and personal fees from Pfizer, personal fees from Arena, personal fees from Avillion, personal fees from Bristol Myers Squibb, personal fees from Boehringer Ingelheim, outside the submitted work.

Prof. Smith reports grants from Abbvie, Sanofi, Novartis, and Pfizer and through consortia with multiple academic partners (psort.org.uk, BIOMAP-IMI.eu), outside the submitted work. 
Dr. Torres reports grants and personal fees from AbbVie, Almirall, Amgen, Arena Pharmaceuticals, Biogen, Biocad, Boehringer Ingelheim, Bristol-Myers Squibb, Celgene, Eli Lilly, Janssen, LEO Pharma, MSD, Novartis, Pfizer, Samsung-Bioepis, Sandoz, during the conduct of the study.

Dr. Waweru is on the Board of the International Federation of Psoriasis Associations who have received grants from Abbvie, Almirall, Amgen, Bristol Meyers Squibb, Boehringer Ingelheim, Celgene, Janssen, Leo Pharma, Eli Lilly, Novartis, Sun Pharma, Pfizer, and UCB, outside the submitted work.

Mr. Urmston reports grants from Almirall, grants from Abbvie, grants from Amgen, grants from Celgene, grants from Dermal Laboratories, grants from Eli Lilly, grants from Janssen, grants from LEO Pharma, grants from T and R Derma, grants from UCB, outside the submitted work.

Ms. McAteer reports grants from Abbvie, grants from Almirall, grants from Amgen, grants from Celgene, grants from Dermal Laboratories, grants from Eli Lilly, grants from Janssen, grants from LEO Pharma, grants from UCB, grants from T and R Derma, outside the submitted work.

Prof. Spuls has done consultancies in the past for Sanofi 111017 and AbbVie 041217 (unpaid), received a departmental independent research grant for TREAT NL registry LeoPharma December 2019; is involved in performing clinical trials with many pharmaceutical industries that manufacture drugs used for the treatment of diseases such as psoriasis and atopic dermatitis, for which financial compensation is paid to the department/hospital; and is chief investigator of the systemic and phototherapy atopic eczema registry (TREAT NL) for adults and children, as well as one of the main investigators of the SECURE-AD registry.

\section{Figure legends}

Fig 1. COVID-19 vaccine hesitancy in individuals with psoriasis: (A) overall count, (B) by age and ethnicity (age $<31$ years $n=93,31-50$ years $n=219,51-70$ years $n=261,>70$ years $\mathrm{n}=58$; white ethnicity $\mathrm{n}=559$, non-white ethnicity $\mathrm{n}=70),(\mathrm{C})$ by systemic immunosuppressant therapy (no therapy $n=406$, standard therapy $n=110$, targeted therapy $n=207$ ), (D) association between disrupted access to psoriasis care and COVID-19 vaccine hesitancy. 
(A) Overall

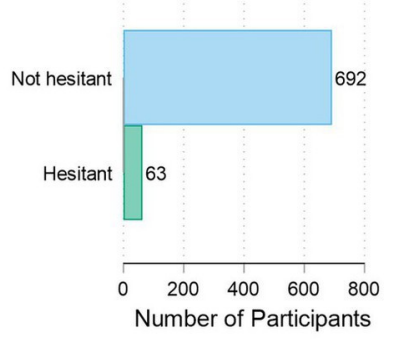

Ethnicity

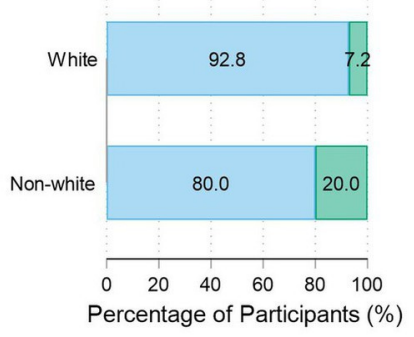

(B) Age

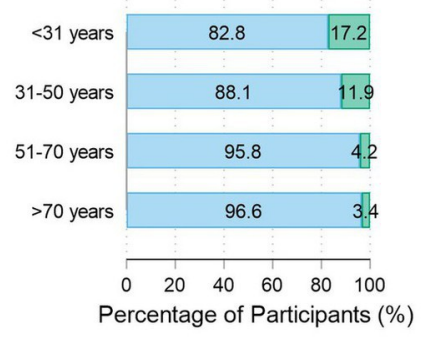

(C) Systemic Therapy

Not hesitant

$\square$ Hesitant or not having vaccine

(D) Association between disrupted access to psoriasis care and COVID-19 vaccine hesitancy
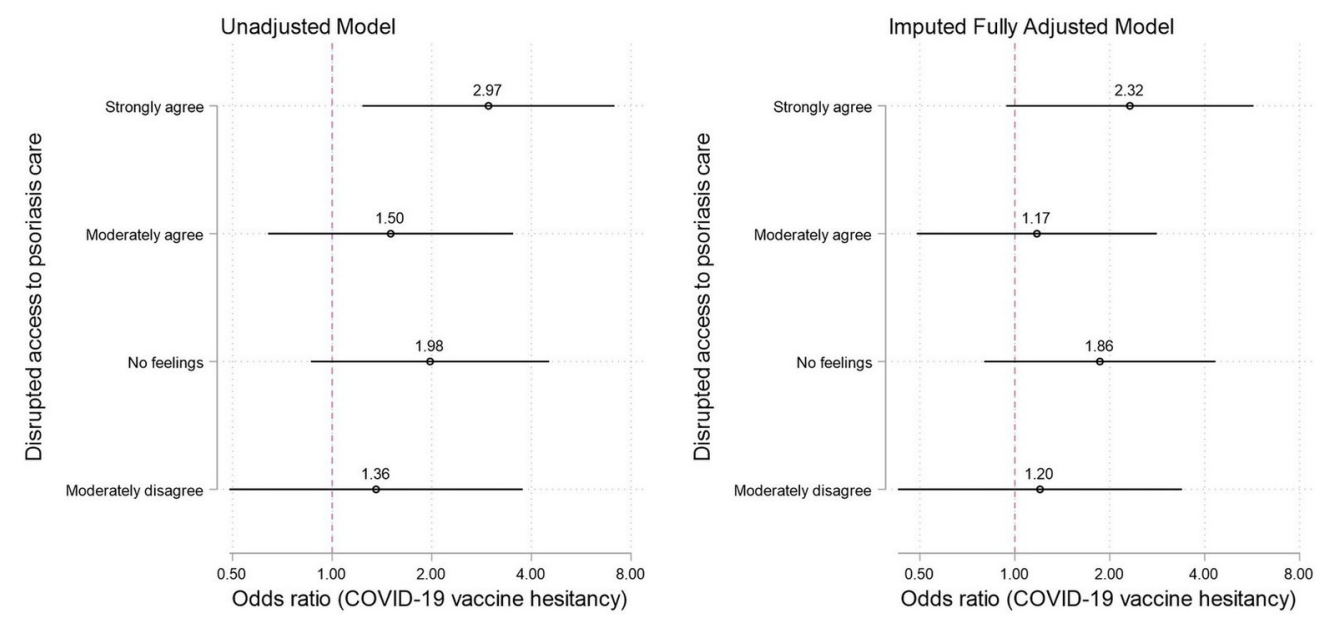

Reference group: 'strongly disagree'

BJD_21042_Figure 1.jpg 